\title{
A new definition of conformable fractional derivative on arbitrary time scales
}

\author{
Mohamad Rafi Segi Rahmat ${ }^{1 *}$ (1)
}

\section{"Correspondence:}

Mohd.Rafi@nottingham.edu.my

'School of Applied Mathematics,

The University of Nottingham

Malaysia, Semenyih, Malaysia

\begin{abstract}
In this paper, a new kind of conformable fractional derivative on arbitrary time scales is introduced. The basic conformable derivative rules are proved. We introduce a new definition of exponential functions, and their potential uses in the definition of conformable integrations are explored. Linear first-order conformable differential equations with constant coefficients are investigated, as well as the conformable analogue of Gronwall's inequality.
\end{abstract}

Keywords: Conformable derivatives; Conformable calculus; Delta derivatives; Time scale calculus; Fractional calculus

\section{Introduction}

A new definition (Definition 1.1) of conformable derivative was introduced and explored in [1]. The basic principle of this new definition is the use of proportional-derivative (PD) controller in control theory where $u$ and time $t$ with two tuning parameters have the algorithm

$$
u(t)=\kappa_{p} E(t)+\kappa_{d} \frac{d}{d t} E(t)
$$

where $\kappa_{p}$ and $\kappa_{d}$ are the proportional gain and derivative gain, respectively, and $E$ is the error between the state variable and the process variable. As a result, the following definition was introduced.

Definition 1.1 ([1]) Let $\alpha \in[0,1]$, and let the functions $\kappa_{0}, \kappa_{1}:[0,1] \times \mathbb{R} \rightarrow \mathbb{R}_{0}^{+}$(where $\left.\mathbb{R}_{0}^{+}=[0, \infty)\right)$ be continuous such that

$$
\begin{aligned}
& \lim _{\alpha \rightarrow 0^{+}} \kappa_{0}(\alpha, t)=0, \quad \lim _{\alpha \rightarrow 0^{+}} \kappa_{1}(\alpha, t)=1, \\
& \lim _{\alpha \rightarrow 1^{-}} \kappa_{0}(\alpha, t)=1, \quad \lim _{\alpha \rightarrow 1^{-}} \kappa_{1}(\alpha, t)=0, \\
& \kappa_{0}(\alpha, t) \neq 0, \quad \alpha \in(0,1], \quad \kappa_{1}(\alpha, t) \neq 0, \quad \alpha \in[0,1) .
\end{aligned}
$$

Then the following differential operator $D^{\alpha}$, defined via

$$
D^{\alpha} f(t)=\kappa_{1}(\alpha, t) f(t)+\kappa_{0}(\alpha, t) f^{\prime}(t)
$$

is conformable provided the function $f$ is differentiable at $t$ and $f^{\prime}:=\frac{d}{d t} f$.

(c) The Author(s) 2019. This article is distributed under the terms of the Creative Commons Attribution 4.0 International License (http://creativecommons.org/licenses/by/4.0/), which permits unrestricted use, distribution, and reproduction in any medium, provided you give appropriate credit to the original author(s) and the source, provide a link to the Creative Commons license, and indicate if changes were made. 
For example, one could choose $\kappa_{1} \equiv(1-\alpha) \omega^{\alpha}$ and $\kappa_{0} \equiv \alpha \omega^{1-\alpha}$ for any $\omega \in(0, \infty)$, or $\kappa_{1} \equiv(1-\alpha)|t|^{\alpha}$ and $\kappa_{0} \equiv \alpha|t|^{1-\alpha}$ for any $\mathbb{R} \backslash\{0\}$, so that

$$
D^{\alpha} f(t)=(1-\alpha)|t|^{\alpha} f(t)+\alpha|t|^{1-\alpha} f^{\prime}(t)
$$

The new definition provided in Definition 1.1 satisfies some of the agreed properties of fractional derivatives such as $D^{0} f(t)=f(t)$ and $D^{1} f(t)=\frac{d}{d t} f(t)$. It is best to consider the conformable derivative in its own right, independent of existing fractional derivative theory.

Definition 1.2 ([1]) Let $\alpha \in[0,1]$. A differential operator $D^{\alpha}$ is conformable if and only if $D^{0}$ is an identity operator and $D^{1}$ is the classical differential operator.

In [1], the authors also explored results including basic conformable derivative and integral rules, conformable exponential functions, Taylor's theorem, reduction of order, variation of parameters, complete characterization of solutions for constant coefficient and Cauchy-Euler type conformable equations, Cauchy functions, variation of constants, a self-adjoint equation, and Sturm-Liouville problems.

In time scales settings, several definitions of conformable fractional derivatives have been introduced. Interestingly, in [2], a conformable fractional derivative operator $\mathbf{T}_{\alpha}$, where $\alpha \in(0,1]$, is defined via

$$
\left|[f(\sigma(t))-f(s)] t^{1-\alpha}-\mathbf{T}_{\alpha}(f)(t)[\sigma(t)-s]\right| \leq \epsilon|\sigma(t)-s|
$$

and in [3], we have

$$
\left|[f(\sigma(t))-f(s)]-\mathbf{T}_{\alpha}\left(f^{\Delta}\right)(t)\left[\sigma(t)^{\alpha}-s^{\alpha}\right]\right| \leq \epsilon\left|\sigma(t)^{\alpha}-s^{\alpha}\right| .
$$

In both definitions, it is obvious that $\mathbf{T}_{0}(f) \neq f$, and it is not conformable according to Definition 1.2. There are several follow-up papers using at least one of the above conformable definitions, including [3-9]. The authors in [1] have also mentioned the possible extension of conformation derivatives on time scales (see [1], Remark 1.5) via

$$
D^{\alpha} f(t)=\kappa_{1}(\alpha, t) f(t)+\kappa_{0}(\alpha, t) f^{\Delta}(t)
$$

With this in mind, in Sect. 2, we introduce and explore a generalization of conformable $\Delta$ - derivative of order $\alpha$ where $0 \leq \alpha \leq 1$ on an arbitrary time scale $\mathbb{T}$ which includes Definition 1.1 as a particular case when $\mathbb{T}=\mathbb{R}$. Fundamental results, e.g., the product rule and the quotient rule, are presented. In Sect. 3 , we introduce the conformable $\Delta$-exponential functions. We derive some important properties of the conformable $\Delta$-exponential functions that are required in the following sections. In Sect. 4, we introduce and explore a version of conformable $\Delta$-integral defined via the conformable exponential function. We also derive analogues of derivative of integral and integration by parts. Section 5 is concerned with conformable linear dynamic equations and dynamic inequalities. A variation of constants formula and an analogue of Gronwall's inequality are presented. 


\section{Conformable delta derivative}

In this section we introduce the generalized conformable derivative on arbitrary time scales $\mathbb{T}$ which also includes Definition 1.1. The readers interested in the theory of time scales are referred to the books $[10,11]$ and articles [12-14]. Here we only provide the necessary concepts of the time scales required for this study.

A time scale $\mathbb{T}$ is a nonempty, closed subset of $\mathbb{R}$ equipped with topology induced from the standard topology on $\mathbb{R}$. For $t \in \mathbb{T}$, the forward and backward jump operators $\sigma, \rho$ : $\mathbb{T} \rightarrow \mathbb{T}$ are respectively defined via

$$
\sigma(t):=\inf \{s \in \mathbb{T}: s>t\}, \quad \rho(t)=\sup \{s \in \mathbb{T}: s<t\} .
$$

In this definition we put $\inf \emptyset=\sup \mathbb{T}$ and $\sup \emptyset=\inf \mathbb{T}$. If $\sigma(t)>t$, we say that $t$ is rightscattered, while if $\rho(t)<t$, we say that $t$ is left-scattered. If $\rho(t)<t<\sigma(t)$, then $t$ is isolated. On the other hand, if $t<\sup \mathbb{T}$ and $\sigma(t)=t$, then $t$ is called right-dense, and if $t>\inf \mathbb{T}$ and $\rho(t)=t$, then $t$ is called left-dense. If $\rho(t)=t=\sigma(t)$, then $t$ is dense.

The graininess function $\mu: \mathbb{T} \rightarrow[0, \infty)$ is defined by

$$
\mu(t):=\sigma(t)-t
$$

We also need the set $\mathbb{T}^{\kappa}$ which is derived from the time scale $\mathbb{T}$. If $\mathbb{T}$ has a left-scattered maximum $m$, then $\mathbb{T}^{\kappa}=\mathbb{T}-\{m\}$. Otherwise, $\mathbb{T}^{\kappa}=\mathbb{T}$.

Definition 2.1 ([10]) A function $f: \mathbb{T} \rightarrow \mathbb{R}$ is called rd-continuous provided it is continuous at right-dense points in $\mathbb{T}$ and its left-side limits exist (finite) at left-dense points in $\mathbb{T}$.

The set of rd-continuous functions $f: \mathbb{T} \rightarrow \mathbb{R}$ will be denoted by $C_{r d}(\mathbb{T})$.

Definition $2.2([10])$ Assume that $f: \mathbb{T} \rightarrow \mathbb{R}$ is a function, and let $t \in \mathbb{T}^{\kappa}$. Let $f^{\Delta}(t)$ be a real number (provided it exists) with the property that for any $\epsilon>0$, there exists a neighborhood $\mathcal{U}$ of $t$ such that

$$
\left|[f(\sigma(t))-f(s)]-f^{\Delta}(t)[\sigma(t)-s]\right| \leq \epsilon|\sigma(t)-s| \quad \forall s \in \mathcal{U}
$$

We call $f^{\Delta}(t)$ the $\Delta$-derivative of $f$ at $t$.

It follows that if $f: \mathbb{T} \rightarrow \mathbb{R}$ is continuous at $t$ and $t$ is right-scattered, then

$$
f^{\Delta}(t)=\frac{f(\sigma(t))-f(t)}{\mu(t)} .
$$

If $t$ is right-dense, then

$$
f^{\Delta}(t)=\lim _{s \rightarrow t} \frac{f(t)-f(s)}{t-s} .
$$

Theorem 2.3 ([10]) Assume that $f, g: \mathbb{T} \rightarrow \mathbb{R}$ are differentiable at $t \in \mathbb{T}^{\kappa}$. Then

(i) $(f+g)^{\Delta}(t)=f^{\Delta}(t)+g^{\Delta}(t)$. 
(ii) For any constant $k,(k f)^{\Delta}(t)=k f^{\Delta}(t)$.

(iii) (Product rule) $(f g)^{\Delta}(t)=f^{\Delta}(t) g(t)+f^{\sigma}(t) g^{\Delta}(t)$.

(iv) If $f(t) f^{\sigma}(t) \neq 0$, then $(1 / f)^{\Delta}=-\frac{f^{\Delta}(t)}{f(t) f^{\sigma}(t)}$.

(v) If $g(t) g^{\sigma}(t) \neq 0$, then $(f / g)^{\Delta}=\frac{f^{\Delta}(t) g(t)-f(t) g^{\Delta}(t)}{g(t) g^{\sigma}(t)}$.

Theorem 2.4 ([10]) Every rd-continuous function $f$ has an antiderivative $F$, i.e., $F^{\Delta}=f(t)$. In particular, if $s \in \mathbb{T}$, then $F$ defined by

$$
F(t):=\int_{s}^{t} f(\tau) \Delta \tau \quad \forall t \in \mathbb{T}
$$

is an antiderivative of $f$, i.e.,

$$
\left(\int_{s}^{t} f(\tau) \Delta \tau\right)^{\Delta}=f(t)
$$

Hence,

$$
\int_{t}^{\sigma(t)} f(t) \Delta t=\mu(t) f(t)
$$

Theorem 2.5 ([10]) Let $s \in \mathbb{T}^{\kappa}$ and assume $f: \mathbb{T} \times \mathbb{T}^{\kappa} \rightarrow \mathbb{R}$ is continuous at $(t, t)$, where $t \in \mathbb{T}^{\kappa}$ with $t>s$. Then

$$
\left(\int_{s}^{t} f(t, \tau) \Delta \tau\right)^{\Delta_{t}}=\int_{s}^{t} f^{\Delta}(t, \tau) \Delta \tau+f(\sigma(t), t),
$$

where $\Delta_{t}$ denotes the delta derivative with respect to $t$.

Now we consider a function $f: \mathbb{T} \rightarrow \mathbb{R}$ and define the so-called conformable delta derivative of $f$ of order $\alpha \in[0,1]$ at a point $t \in \mathbb{T}^{\kappa}$.

Remark 2.6 The proofs of theorems and lemmas in this section and the following sections are subject to the left-scattered points on $\mathbb{T}$ only. For the left-dense point (i.e. the case $\mu(t)=0)$, the proofs are given in [1], so we omit them in this paper.

Definition 2.7 (The conformable $\Delta$-derivative) Assume that $f: \mathbb{T} \rightarrow \mathbb{R}$ is a function, $t \in \mathbb{T}^{\kappa}$, and $\kappa_{0}, \kappa_{1}$ are continuous and satisfy (1). Let $\Delta^{\alpha} f(t), \alpha \in[0,1]$ be a real number (provided it exists) with the property that for any $\epsilon>0$, there exists a neighborhood $\mathcal{U}$ of $t$ such that

$$
\begin{aligned}
& \left|\kappa_{1}(\alpha, t) f(t)[\sigma(t)-s]+\kappa_{0}(\alpha, t)[f(\sigma(t))-f(s)]-\left(\Delta^{\alpha} f\right)(t)[\sigma(t)-s]\right| \\
& \quad \leq \epsilon[\sigma(t)-s] \quad \text { for all } s \in \mathcal{U} .
\end{aligned}
$$

We call $\Delta^{\alpha} f(t)$ the conformable $\Delta$-derivative of $f$ of order $\alpha$ at $t$. 
Example 2.8 Let $\alpha \in[0,1]$.

(i) Let $f: \mathbb{T} \rightarrow \mathbb{R}$ be defined by $f(t)=c$ for all $t \in \mathbb{T}$, where $c \in \mathbb{R}$ is constant. Then by (9) we have

$$
\begin{aligned}
& \left|c \kappa_{1}(\alpha, t)-\left(\Delta^{\alpha} f\right)(t)\right| \leq \epsilon \\
& \quad \Rightarrow \quad\left(\Delta^{\alpha} f\right)(t)=c \kappa_{1}(\alpha, t), \quad \forall t \in \mathbb{T} .
\end{aligned}
$$

(ii) Let $f: \mathbb{T} \rightarrow \mathbb{R}$ be defined by $f(t)=t$ for all $t \in \mathbb{T}$, then by (9) again we get

$$
\begin{aligned}
& \left|t \kappa_{1}(\alpha, t)+\kappa_{0}(\alpha, t)-\left(\Delta^{\alpha} f\right)(t)\right| \leq \epsilon \\
& \quad \Rightarrow \quad \Delta^{\alpha} f(t)=\kappa_{0}(\alpha, t)+t \kappa_{1}(\alpha, t), \quad \forall t \in \mathbb{T} .
\end{aligned}
$$

The following theorem implicates the useful relationships concerning the conformable delta derivative. Let us denote the set of all conformable delta differentiable functions by

$$
\Phi(\mathbb{T}):=\left\{f: \mathbb{T} \rightarrow \mathbb{R}: \Delta^{\alpha} f(t) \text { exists and finite for all } t \in \mathbb{T}^{\kappa}\right\}
$$

Theorem 2.9 Let $\mathbb{T}$ be a time scale. Assume that $f: \mathbb{T} \rightarrow \mathbb{R}$, and let $t \in \mathbb{T}^{\kappa}$. The following properties hold.

(i) If $f \in \Phi(\mathbb{T})$, then $f$ is continuous at $t$.

(ii) If $f$ is continuous at $t$ and $t$ is right-scattered, then $f \in \Phi(\mathbb{T})$ if and only if

$$
f^{\Delta}(t)=\frac{f(\sigma(t))-f(t)}{\sigma(t)-t}
$$

exists. In this case, we write

$$
\Delta^{\alpha} f(t)=\kappa_{0}(\alpha, t) f^{\Delta}(t)+\kappa_{1}(\alpha, t) f(t)
$$

(iii) If $t$ is right-dense, then $f \in \Phi(\mathbb{T})$ if and only if, the limit

$$
\lim _{t \rightarrow s} \frac{f(t)-f(s)}{t-s}=f^{\prime}(t)
$$

exists as a finite number. In this case, we write

$$
\Delta^{\alpha} f(t)=\kappa_{0}(\alpha, t) f^{\prime}(t)+\kappa_{1}(\alpha, t) f(t)
$$

Proof The proof is similar to Theorem 1.16 in [10]. Thus, we omit them.

Next, we would like to be able to find the conformable delta derivatives of sum, product, and quotients of delta differentiable functions in $\mathbb{T}$.

Lemma 2.10 Assume that $f, g: \mathbb{T} \rightarrow \mathbb{R}$ are conformable delta-differentiable at $t \in \mathbb{T}^{\kappa}$ and $\kappa_{0}, \kappa_{1}$ are continuous and satisfy (1). Then

(i) $\Delta^{\alpha}[a f+b g]=a \Delta^{\alpha}[f]+b \Delta^{\alpha}[g]$ for all $a, b \in \mathbb{R}$;

(ii) $\Delta^{\alpha}[f g]=f^{\sigma} \cdot \Delta^{\alpha}[g]+\Delta^{\alpha}[f] \cdot g-f^{\sigma} \cdot g \kappa_{1}(\alpha, \cdot)$; 
(iii) $\Delta^{\alpha}[1 / g]=-\frac{\Delta^{\alpha} g}{g \cdot g^{\sigma}}+\left(\frac{1}{g}+\frac{1}{g^{\sigma}}\right) k_{1}$, provided $g g^{\rho} \neq 0$;

(iv) $\Delta^{\alpha}[f / g]=\frac{\Delta^{\alpha}[f] \cdot g^{\sigma}-f \cdot \Delta^{\alpha}[g]}{g \cdot g^{\sigma}}+\frac{f^{\sigma}}{g^{\sigma}} \kappa_{1}(\alpha, \cdot)$, provided $g g^{\rho} \neq 0$.

Proof Item (i) can be easily proved using formula (9). For (ii), we use the product rule

$$
[f g]^{\Delta}=f^{\Delta} g+f^{\sigma} g^{\Delta}=f g^{\Delta}+f^{\Delta} g^{\sigma} .
$$

From (10), we have

$$
\begin{aligned}
\Delta^{\alpha}[f g] & =\kappa_{0}[f g]^{\Delta}+\kappa_{1}[f g]=\kappa_{0}\left[f^{\Delta} g+f^{\sigma} g^{\Delta}\right]+\kappa_{1}[f g] \\
& =\left[\kappa_{1} f+\kappa_{0} f^{\Delta}\right] g+f^{\sigma}\left[\kappa_{1} g+\kappa_{0} g^{\Delta}\right]-\kappa_{1} f^{\sigma} g \\
& =\Delta^{\alpha}[f] g+f^{\sigma} \Delta^{\alpha}[g]-\kappa_{1} f^{\sigma} g .
\end{aligned}
$$

To prove (iii), using the fact $\Delta^{\alpha}[1]=\kappa_{1}(\alpha, t)$ from Example 2.8(i)

$$
\Delta^{\alpha}[g \cdot 1 / g]=\Delta^{\alpha}[1]=\kappa_{1}(\alpha, \cdot) .
$$

Hence, using (ii) above,

$$
\begin{aligned}
& \Delta^{\alpha}[g \cdot 1 / g]=g \cdot \Delta^{\alpha}[1 / g]+\Delta^{\alpha}[g] .\left(1 / g^{\sigma}\right)-\kappa_{1} \cdot g / g^{\sigma}=\kappa_{1}(\alpha, \cdot), \\
& g . \Delta^{\alpha}[1 / g]=-\Delta^{\alpha}[g] / g^{\sigma}+\kappa_{1} \cdot g / g^{\sigma}+\kappa_{1}(\alpha, \cdot), \\
& \Delta^{\alpha}[1 / g]=-\Delta^{\alpha}[g] / g \cdot g^{\sigma}+\kappa_{1} 1 / g^{\sigma}+\kappa_{1}(\alpha, \cdot) 1 / g .
\end{aligned}
$$

The proof of (iv) is easily obtained by using (ii) and (iii) in $\Delta^{\alpha}(f \cdot 1 / g)$.

\section{Conformable exponential function}

Now, we give a new definition for conformable exponential function with respect to the operator $\Delta^{\alpha}$ in the time scale $\mathbb{T}$. First we make a preliminary definition.

Definition 3.1 Let $\alpha \in[0,1]$ and the functions $\kappa_{0}, \kappa_{1}:[0,1] \times \mathbb{T} \rightarrow \mathbb{R}_{0}^{+}$be continuous and satisfy (1). We say that a function $p: \mathbb{T} \rightarrow \mathbb{R}$ is $\alpha$-regressive provided

$$
1+\frac{p(\tau)-\kappa_{1}(\alpha, \tau)}{\kappa_{0}(\alpha, \tau)} \mu(\tau) \neq 0 \quad \text { for all } t \in \mathbb{T}^{\kappa}
$$

holds. The set of all $\alpha$-regressive and rd-continuous functions in $\mathbb{T}$ will be denoted by

$$
\mathcal{R}_{\alpha}=\mathcal{R}_{\alpha}(\mathbb{T}) .
$$

Next, we introduce the analogue of "circle minus" on $\mathcal{R}_{\alpha}$.

Definition 3.2 Let $p \in \mathcal{R}_{\alpha}$ and $\alpha \in(0,1]$. We define the "conformable circle minus" $\kappa_{1} \ominus_{\alpha}$ on $\mathcal{R}_{\alpha}$ via

$$
\left(\kappa_{1} \ominus_{\alpha} p\right)(t):=\kappa_{1}(\alpha, t)-\frac{p(t)}{1+\frac{p(t)-\kappa_{1}(\alpha, t)}{\kappa_{0}(\alpha, t)} \mu(t)} \quad \text { for all } t \in \mathbb{T}^{\kappa} .
$$


Remark 3.3 It is clear that for $\alpha \rightarrow 1^{-}$we have

$$
\left(\kappa_{1} \ominus_{\alpha} p\right)(t) \equiv(\ominus p)(t)=-\frac{p(t)}{1+p(t) \mu(t)} \quad \text { for all } t \in \mathbb{T}^{\kappa},
$$

as defined in [10].

Lemma 3.4 Let $p \in \mathcal{R}_{\alpha}$ and $\alpha \in(0,1]$. Then

(i) $\kappa_{1} \ominus_{\alpha} p \in \mathcal{R}_{\alpha}$;

(ii) $\kappa_{1} \ominus_{\alpha}\left(\kappa_{1} \ominus_{\alpha} p\right)=p$.

Proof (i). It is clear that since $p \in \mathcal{R}_{\alpha}$, we have

$$
\kappa_{1}(\alpha, t)-\frac{p(t)}{1+\frac{p(t)-\kappa_{1}(\alpha, t)}{\kappa_{0}(\alpha, t)} \mu(t)} \in \mathcal{R}_{\alpha}
$$

Hence, the claim follows.

(ii) Follows from the definition by substituting $p$ in (12) with $\kappa_{1} \ominus_{\alpha} p$.

The conformable exponential function $\widetilde{e}_{p}(t, s)$ is defined next.

Definition 3.5 Let $\alpha \in(0,1]$ and $p \in \mathcal{R}_{\alpha}$. Let $\kappa_{0}, \kappa_{1}$ be continuous and satisfy (1) with $p / \kappa_{0}$ and $\kappa_{1} / \kappa_{0} \Delta$-integrable on $\mathbb{T}$. Then the conformable exponential function with respect to the operator $\Delta^{\alpha}$ in $\mathbb{T}$ is defined to be

$$
\begin{aligned}
& \tilde{e}_{p}(t, s)=\exp \left[\int_{s}^{t} \frac{1}{\mu(\tau)} \log \left(1+\frac{p(\tau)-\kappa_{1}(\alpha, \tau)}{\kappa_{0}(\alpha, \tau)} \mu(\tau)\right) \Delta \tau\right], \\
& \widetilde{e}_{0}(t, s)=\exp \left[\int_{s}^{t} \frac{1}{\mu(\tau)} \log \left(1-\frac{\kappa_{1}(\alpha, \tau)}{\kappa_{0}(\alpha, \tau)} \mu(\tau)\right) \Delta \tau\right]
\end{aligned}
$$

for all $s, t \in \mathbb{T}$, where $\log$ is the principle logarithm function. For $\mu(t)=0$, we define

$$
\tilde{e}_{p}(t, s)=\exp \left[\int_{s}^{t}\left(\frac{p(\tau)-\kappa_{1}(\alpha, \tau)}{\kappa_{0}(\alpha, \tau)}\right) \Delta \tau\right], \quad \widetilde{e}_{0}(t, s)=\exp \left[-\int_{s}^{t} \frac{\kappa_{1}(\alpha, \tau)}{\kappa_{0}(\alpha, \tau)} \Delta \tau\right]
$$

as defined in [1]. Note that the conformable exponential function $\widetilde{e}_{p}(t, s)$ is consistent with exponential function $e_{z}(t, s)$ defined in the arbitrary time scales [10], where we can assume $z=\frac{p-\kappa_{1}}{\kappa_{0}}$.

Definition 3.6 Let $p: \mathbb{T} \rightarrow \mathbb{R}$ and $\alpha \in(0,1]$. Define the set $\mathcal{R}_{\alpha}^{+}$of all positively $\alpha$-regressive elements of $\mathcal{R}_{\alpha}$ via

$$
\mathcal{R}_{\alpha}^{+}=\left\{p \in \mathcal{R}_{\alpha}: 1+\frac{p(\tau)-\kappa_{1}(\alpha, \tau)}{\kappa_{0}(\alpha, \tau)} \mu(\tau)>0 \text { for all } t \in \mathbb{T}\right\}
$$

Lemma 3.7 Let $p \in \mathbb{R}, \alpha \in(0,1]$ and $t_{0} \in \mathbb{T}$. If $p \in \mathcal{R}_{\alpha}^{+}$, then $\widetilde{e}_{p}\left(t, t_{0}\right)>0$ for all $t \in \mathbb{T}$. 
Proof Since $1+\frac{p(\tau)-\kappa_{1}(\alpha, \tau)}{\kappa_{0}(\alpha, \tau)} \mu(\tau)>0$, this implies that

$$
\frac{1}{\mu(t)} \log \left(1+\frac{p(\tau)-\kappa_{1}(\alpha, \tau)}{\kappa_{0}(\alpha, \tau)} \mu(\tau)\right) \in \mathbb{R} \quad \text { for all } t \in \mathbb{R}^{\kappa}
$$

Hence $\widetilde{e}_{p}\left(t, t_{0}\right)>0$ for all $t \in \mathbb{T}$, by formula (13). This completes the proof.

We proceed by collecting some important properties of the conformable exponential function.

Theorem 3.8 If $p \in \mathcal{R}_{\alpha}^{+}$and $\alpha \in(0,1]$, then

(i) $\widetilde{e}_{p}(\sigma(t), s)=\left(1+\frac{p(t)-\kappa_{1}(\alpha, t)}{\kappa_{0}(\alpha, t)} \mu(t)\right) \widetilde{e}_{p}(t, s)$;

(ii) $\frac{\widetilde{e}_{0}(t, s)}{\widetilde{e}_{p}(t, s)}=\widetilde{e}_{\kappa_{1} \ominus_{\alpha} p}(t, s)$;

(iii) $\widetilde{e}_{p}(t, s)=\frac{1}{\widetilde{e}_{p}(s, t)}$;

(iv) $\widetilde{e}_{p}(t, s) \widetilde{e}_{p}(s, r)=\widetilde{e}_{p}(t, r)$;

(v) $\widetilde{e}_{p}^{\Delta}(t, s)=\left(\frac{p(t)-\kappa_{1}(\alpha, t)}{\kappa_{0}(\alpha, t)}\right) \widetilde{e}_{p}(t, s)$;

(vi) $\left(\frac{1}{\widetilde{e}_{p}(t, s)}\right)^{\Delta}=-\left(\frac{p(t)-\kappa_{1}(\alpha, t)}{\kappa_{0}(\alpha, t)}\right) \frac{1}{\widetilde{e}_{p}(\sigma(t), s)}$.

Proof (i) Since $\widetilde{e}_{p}(\sigma(t), s)=\widetilde{e}_{p}(t, s) \cdot \widetilde{e}_{p}(\sigma(t), t)$, the result follows from (13).

(ii) Obtained by substituting $\left(\kappa_{1} \ominus_{\alpha} p\right)$ into (13).

(iii) By Eq. (13), it follows that $\widetilde{e}_{p}(t, s)=\frac{1}{\widetilde{e}_{p}(s, t)}$.

(iv) It is straightforward by definition (13).

(v) By use of delta derivative (5) we get

$$
\begin{aligned}
\widetilde{e}_{p}^{\Delta}(t, s) & =\frac{\widetilde{e}_{p}(\sigma(t), s)-\widetilde{e}_{p}(t, s)}{\mu(t)} \\
& =\frac{\widetilde{e}_{p}(t, s) \widetilde{e}_{p}(\sigma(t), t)-\widetilde{e}_{p}(t, s)}{\mu(t)} \\
& =\frac{\widetilde{e}_{p}(\sigma(t), t)-1}{\mu(t)} \widetilde{e}_{p}(t, s) \\
& =\frac{1+\frac{p(t)-\kappa_{1}(\alpha, t)}{\kappa_{0}(\alpha, t)} \mu(t)-1}{\mu(t)} \widetilde{e}_{p}(t, s) \\
& =\frac{p(t)-\kappa_{1}(\alpha, t)}{\kappa_{0}(\alpha, t)} \widetilde{e}_{p}(t, s) .
\end{aligned}
$$

(vi) a direct consequence of Theorem 2.3(iv).

Lemma 3.9 For $\alpha \in(0,1]$ and $p \in \mathcal{R}_{\alpha}$. For fixed $s \in \mathbb{T}$, the conformable delta exponential function satisfies

(i) $\Delta^{\alpha}\left[\widetilde{e}_{p}(\cdot, s)\right]=p(t) \widetilde{e}_{p}(\cdot, s)$,

(ii) $\Delta^{\alpha}\left[\widetilde{e}_{\kappa_{1} \ominus_{\alpha} p}(\cdot, s)\right]=\left[\kappa_{1}(\alpha, \cdot)-p\right]\left[\widetilde{e}_{\kappa_{1} \ominus_{\alpha} p}(\cdot, s)\right]^{\sigma}$.

Proof (i). By Theorem 3.8(v) we have

$$
\Delta^{\alpha}\left[\widetilde{e}_{p}(t, s)\right]=\kappa_{1}(\alpha, t) \widetilde{e}_{p}(t, s)+\kappa_{0}(\alpha, t) \widetilde{e}_{p}^{\Delta}(t, s)
$$




$$
\begin{aligned}
\Delta^{\alpha}\left[\widetilde{e}_{p}(t, s)\right] & =\kappa_{1}(\alpha, t) \widetilde{e}_{p}(t, s)+\kappa_{0}(\alpha, t) \frac{p(\tau)-\kappa_{1}(\alpha, t)}{\kappa_{0}(\alpha, t)} \widetilde{e}_{p}(t, s) \\
& =\kappa_{1}(\alpha, t) \widetilde{e}_{p}(t, s)+p(\tau) \widetilde{e}_{p}(t, s)-\kappa_{1}(\alpha, t) \widetilde{e}_{p}(t, s) \\
& =p(t) \widetilde{e}_{p}(t, s) .
\end{aligned}
$$

(ii) From (i) we have

$$
\begin{aligned}
\Delta^{\alpha}\left[\widetilde{e}_{\kappa_{1} \ominus_{\alpha} p}(\cdot, s)\right] & =\left(\kappa_{1} \ominus_{\alpha} p\right)(t) \widetilde{e}_{\kappa_{1} \ominus_{\alpha} p}(\cdot, s) \\
& =\left(\kappa_{1}(\alpha, t)-\frac{p(t)}{1+\frac{p(t)-\kappa_{1}(\alpha, t)}{\kappa_{0}(\alpha, t)} \mu(t)}\right) \widetilde{e}_{\kappa_{1} \ominus_{\alpha} p}(t, s) \\
& \left.=\left[\kappa_{1}(\alpha, t)-p(t)\right]\left(\frac{1-\frac{\kappa_{1}}{\kappa_{0}} \mu(t)}{1+\frac{p(t)-\kappa_{1}}{\kappa_{0}} \mu(t)}\right)\right) \widetilde{e}_{0}(t, s) \\
\widetilde{e}_{p}(t, s) & {\left[\kappa_{1}(\alpha, t)-p(t)\right] \frac{\widetilde{e}_{0}(\sigma(t), s)}{\widetilde{e}_{p}(\sigma(t), s)} } \\
& =\left[\kappa_{1}(\alpha, t)-p(t)\right] \widetilde{e}_{\kappa_{1} \ominus_{\alpha} p}(\sigma(t), s) .
\end{aligned}
$$

Hence, the proof is completed.

Lemma 3.10 For $\alpha \in(0,1]$ and for the conformable exponential function $\widetilde{e}_{0}$ as defined in (13), we have

$$
\Delta^{\alpha}\left[\int_{a}^{t} \frac{f(\tau) \widetilde{e}_{0}(t, \sigma(\tau))}{\kappa_{0}(\alpha, \tau)} \Delta \tau\right]=f(t) .
$$

Proof By (10) and Theorem 2.5, we have

$$
\begin{aligned}
\Delta^{\alpha} & {\left[\int_{a}^{t} \frac{f(\tau) \widetilde{e}_{0}(t, \sigma(\tau))}{\kappa_{0}(\alpha, \tau)} \Delta \tau\right] } \\
= & \kappa_{1}(\alpha, t) \int_{a}^{t} \frac{f(\tau) \widetilde{e}_{0}(t, \sigma(\tau))}{\kappa_{0}(\alpha, \tau)} \Delta \tau+\kappa_{0}(\alpha, t)\left(\frac{f(\tau) \widetilde{e}_{0}(t, \sigma(\tau))}{\kappa_{0}(\alpha, \tau)}\right)^{\Delta t} \\
= & \kappa_{1}(\alpha, t) \int_{a}^{t} \frac{f(\tau) \widetilde{e}_{0}(t, \sigma(\tau))}{\kappa_{0}(\alpha, \tau)} \Delta \tau+\kappa_{0}(\alpha, t) \int_{a}^{t} \frac{f(\tau) \widetilde{e}_{0}^{\Delta t}(t, \sigma(\tau))}{\kappa_{0}(\alpha, \tau)} \Delta \tau \\
& +\kappa_{0}(\alpha, t) \frac{f(t) \widetilde{e}_{0}(\sigma(t), \sigma(t))}{\kappa_{0}(\alpha, t)} \\
= & \kappa_{1}(\alpha, t) \int_{a}^{t} \frac{f(\tau) e_{0}(t, \sigma(\tau))}{\kappa_{0}(\alpha, \tau)} \Delta \tau-\kappa_{1}(\alpha, t) \int_{a}^{t} \frac{f(\tau) \widetilde{e}_{0}(t, \sigma(\tau))}{\kappa_{0}(\alpha, \tau)} \Delta \tau+f(t) \\
= & f(t) .
\end{aligned}
$$

This completes the proof.

\section{Conformable delta integral}

In this section, we introduce the conformable delta integral with respect to $\Delta^{\alpha}$ in $\mathbb{T}$. Several important results will be proved. 
Definition 4.1 Assume that $f \in C_{r d}(\mathbb{R})$ and $\alpha \in(0,1]$, and $t_{0} \in \mathbb{T}$. In light of (14), Lemma 3.9(i), and Lemma 3.10, we define the conformable antiderivative via

$$
\int \Delta^{\alpha} f(t) \Delta_{\alpha} \tau=f(t)+\tilde{c}_{0}\left(t, t_{0}\right), \quad \forall t \in \mathbb{T}, c \in \mathbb{R}
$$

Similarly, define the conformable delta integral of $f$ over the time scale interval $[a, b]_{\mathbb{T}}$ as

$$
\int_{a}^{t} f(\tau) \widetilde{e}_{0}(t, \sigma(\tau)) \Delta_{\alpha} \tau:=\int_{a}^{t} \frac{f(\tau) \widetilde{e}_{0}(t, \sigma(\tau))}{\kappa_{0}(\alpha, \tau)} \Delta \tau, \quad \Delta_{\alpha} \tau:=\frac{1}{\kappa_{0}(\alpha, \tau)} \Delta \tau .
$$

To illustrate the conformable delta integral, let us consider the following simple example.

Example 4.2 Let $\mathbb{T}=\mathbb{Z}, \kappa_{0}(\alpha, t) \equiv \alpha \omega_{0}^{1-\alpha}, \kappa_{1} \equiv 0, \omega_{0} \in(0, \infty), \alpha \in(0,1]$, and $f(t)=a^{t}$, where $a \neq 1$ is a constant. We suppose to evaluate

$$
\int a^{t} \Delta_{\alpha} t
$$

Since

$$
\left(\frac{a^{t}}{a-1}\right)^{\Delta}=\frac{a^{t+1}}{a-1}-\frac{a^{t}}{a-1}=\frac{a^{t+1}-a^{t}}{a-1}=a^{t},
$$

for $\kappa_{0}(\alpha, t) \equiv \alpha \omega_{0}^{1-\alpha}$ and $\kappa_{1} \equiv 0, \omega_{0} \in(0, \infty)$ we have

$$
\Delta^{\alpha}\left(\frac{a^{t}}{a-1}\right)=\alpha \omega_{0}^{1-\alpha} a^{t}
$$

Hence, we write

$$
\begin{aligned}
\int a^{t} \Delta_{\alpha} t & =\frac{1}{\alpha \omega_{0}^{1-\alpha}} \int \Delta^{\alpha}\left(\frac{a^{t}}{a-1}\right) \Delta_{\alpha} t \\
& =\frac{a^{t}}{(a-1) \alpha \omega_{0}^{1-\alpha}}+c .
\end{aligned}
$$

Here we use $\widetilde{e}_{0}\left(t, t_{0}\right) \equiv 1$ for $t, t_{0} \in \mathbb{Z}$. It is clear that for $\alpha=1$ the result agrees with the delta integral in the classical sense (see Example 1.72, [10]).

Following from (16) and Lemma 3.10, we have

Lemma 4.3 Let $\alpha \in(0,1]$ and $f, g \in C_{r d}(\mathbb{R})$. Let the functions $\kappa_{0}$ and $\kappa_{1}$ be continuous and satisfy (1). Then

$$
\Delta^{\alpha}\left[\int_{a}^{t} f(\tau) \widetilde{e}_{0}(t, \sigma(\tau)) \Delta_{\alpha} \tau\right]=f(t)
$$

Note that, if $\alpha \rightarrow 1^{-}$, we have $\widetilde{e}_{0}(t, \sigma(\tau))=1$ and $\Delta\left[\int_{a}^{t} f(\tau) \Delta \tau\right]=f(t)$ as in (5).

In the following lemma, we give the version of integration by parts with respect to the operator $\Delta^{\alpha}$. 
Lemma 4.4 Let $f, g \in \Phi(\mathbb{T})$. Then

(i) $\int_{a}^{t} \Delta^{\alpha}[g(\tau)] \widetilde{e}_{0}(t, \sigma(\tau)) \Delta_{\alpha} \tau=\left[g(\tau) \widetilde{e}_{0}(t, \sigma(\tau))\right]_{\tau=a}^{t}$.

(ii) $\int_{a}^{b} f(t) \Delta^{\alpha}[g(t)] \widetilde{e}_{0}(b, \sigma(t)) \Delta_{\alpha} t=\left[f(t) g(t) \widetilde{e}_{0}(b, \sigma(t))\right]_{t=a}^{b}-\int_{a}^{b} g^{\sigma}(t)\left\{\Delta^{\alpha}[f(t)]-\right.$ $\left.\kappa_{1}(\alpha, t) f(t)\right\} \widetilde{e}_{0}(b, \sigma(t)) \Delta_{\alpha} t$.

Proof (i) is a special case of (ii). To prove (ii), we use Lemma 2.10(ii) and the definition of conformable delta integral (16).

Now, we give an analogue of Theorem 2.5 with respect to $\Delta^{\alpha}$.

Lemma 4.5 Let $\alpha \in(0,1]$ and the function $f: \mathbb{T}^{2} \rightarrow \mathbb{R}$ be rd-continuous in its first variable. Let the functions $\kappa_{0}$ and $\kappa_{1}$ be continuous and satisfy (1). Then

$$
\begin{aligned}
\Delta^{\alpha} & {\left[\int_{a}^{t} f(t, \tau) \widetilde{e}_{0}(t, \sigma(\tau)) \Delta_{\alpha} \tau\right] } \\
& =\int_{a}^{t}\left[\Delta_{t}^{\alpha} f(t, \tau)-\kappa_{1}(\alpha, t) f(t, \tau)\right] \widetilde{e}_{0}(t, \sigma(\tau)) \Delta_{\alpha} \tau+f(\sigma(t), t),
\end{aligned}
$$

or, if $\widetilde{e}_{0}$ is absent,

$$
\Delta^{\alpha}\left[\int_{a}^{t} f(t, \tau) \Delta_{\alpha} \tau\right]=\int_{a}^{t} \Delta_{t}^{\alpha} f(t, \tau) \Delta_{\alpha} \tau+f(\sigma(t), t) .
$$

Proof First note that by Theorem 2.5 we have

$$
\begin{aligned}
& \left(\int_{a}^{t} f(t, \tau) \widetilde{e}_{0}(t, \sigma(\tau)) \Delta_{\alpha} \tau\right)^{\Delta_{t}} \\
& \quad=\int_{a}^{t} \frac{\left[f(t, \tau) \widetilde{e}_{0}(t, \sigma(\tau))\right]^{\Delta t}}{\kappa_{0}(\alpha, \tau)} \Delta \tau+\frac{f(\sigma(t), t)}{\kappa_{0}(\alpha, t)} \\
& \quad=\int_{a}^{t} \frac{\widetilde{e}_{0}(t, \sigma(\tau))}{\kappa_{0}(\alpha, \tau)}\left[f^{\Delta}(t, \tau)-\frac{\kappa_{1}(\alpha, t)}{\kappa_{0}(\alpha, t)} f(\sigma(t), \tau)\right] \Delta \tau+\frac{f(\sigma(t), t)}{\kappa_{0}(\alpha, t)} .
\end{aligned}
$$

Hence, it follows from (11) that

$$
\begin{aligned}
\Delta^{\alpha} & {\left[\int_{a}^{t} f(t, \tau) \widetilde{e}_{0}(t, \sigma(\tau)) \Delta_{\alpha} \tau\right] } \\
= & \kappa_{1}(\alpha, t) \int_{a}^{t} f(t, \tau) \widetilde{e}_{0}(t, \sigma(\tau)) \Delta_{\alpha} \tau+\kappa_{0}(\alpha, t)\left(\int_{a}^{t} f(t, \tau) \widetilde{e}_{0}(t, \sigma(\tau)) \Delta_{\alpha} \tau\right)^{\Delta_{t}} \\
= & \kappa_{1}(\alpha, t) \int_{a}^{t} \frac{f(t, \tau) \widetilde{e}_{0}(t, \sigma(\tau))}{\kappa_{0}(\alpha, \tau)} \Delta \tau \\
& +\kappa_{0}(\alpha, t)\left(\int_{a}^{t} \frac{\widetilde{e}_{0}(t, \sigma(\tau))}{\kappa_{0}(\alpha, \tau)}\left[f^{\Delta}(t, \tau)-\frac{\kappa_{1}(\alpha, t)}{\kappa_{0}(\alpha, t)} f(\sigma(t), \tau)\right] \Delta \tau+\frac{f(\sigma(t), t)}{\kappa_{0}(\alpha, t)}\right) \\
= & \int_{a}^{t} \frac{\widetilde{e}_{0}(t, \sigma(\tau))}{\kappa_{0}(\alpha, \tau)}\left[\kappa_{1}(\alpha, t) f(t, \tau)+\kappa_{0}(\alpha, t) f^{\Delta}(t, \tau)-\kappa_{1}(\alpha, t) f(\sigma(t), \tau)\right] \Delta \tau+f(\sigma(t), t) \\
= & \int_{a}^{t} \frac{\widetilde{e}_{0}(t, \sigma(\tau))}{\kappa_{0}(\alpha, \tau)}\left[\Delta_{t}^{\alpha} f(t, \tau)-\kappa_{1}(\alpha, t) f(\sigma(t), \tau)\right] \Delta \tau+f(\sigma(t), t)
\end{aligned}
$$




$$
=\int_{a}^{t}\left[\Delta_{t}^{\alpha} f(t, \tau)-\kappa_{1}(\alpha, t) f(\sigma(t), \tau)\right] \widetilde{e}_{0}(t, \sigma(\tau)) \Delta_{\alpha} \tau+f(\sigma(t), t) .
$$

For the second expression (19), if $\widetilde{e}_{0}(t, \sigma(\tau))$ is absent from the integral expression, then

$$
\begin{aligned}
\Delta^{\alpha}\left[\int_{a}^{t} f(t, \tau) \Delta_{\alpha} \tau\right] & =\Delta^{\alpha}\left[\int_{a}^{t} \frac{f(t, \tau)}{\kappa_{0}(\alpha, \tau)} \Delta \tau\right] \\
& =\kappa_{1}(\alpha, t) \int_{a}^{t} \frac{f(t, \tau)}{\kappa_{0}(\alpha, \tau)} \Delta \tau+\kappa_{0}(\alpha, t)\left(\int_{a}^{t} \frac{f(t, \tau)}{\kappa_{0}(\alpha, \tau)} \Delta \tau\right)^{\Delta_{t}} \\
& =\kappa_{1}(\alpha, t) \int_{a}^{t} \frac{f(t, \tau)}{\kappa_{0}(\alpha, \tau)} \Delta \tau+\kappa_{0}(\alpha, t)\left[\int_{a}^{t} \frac{f^{\Delta_{t}}(t, \tau)}{\kappa_{0}(\alpha, \tau)} \Delta \tau+\frac{f(\sigma(t), t)}{\kappa_{0}(\alpha, t)}\right] \\
& =\kappa_{1}(\alpha, t) \int_{a}^{t} \frac{f(t, \tau)}{\kappa_{0}(\alpha, \tau)} \Delta \tau+\kappa_{0}(\alpha, t) \int_{a}^{t} \frac{f^{\Delta_{t}}(t, \tau)}{\kappa_{0}(\alpha, \tau)} \Delta \tau+f(\sigma(t), t) \\
& =\int_{a}^{t}\left[\kappa_{1}(\alpha, t) f(t, \tau)+\kappa_{0}(\alpha, t) f^{\Delta_{t}}(t, \tau)\right] \Delta_{\alpha} \tau+f(\sigma(t), t) \\
& =\int_{a}^{t} \Delta_{t}^{\alpha} f(t, \tau) \Delta_{\alpha} \tau+f(\sigma(t), t) .
\end{aligned}
$$

This completes the proof.

\section{Conformable dynamic equations}

In this section, we consider the conformable linear dynamic equations and inequalities on arbitrary time scales. Throughout we let $\alpha \in(0,1]$, and $\kappa_{0}, \kappa_{1}$ satisfy (1). To begin with, let

$$
y=\widetilde{e}_{p}\left(\cdot, t_{0}\right)
$$

for some fixed $t_{0} \in \mathbb{T}$. It is clear that $y\left(t_{0}\right)=\widetilde{e}_{p}\left(t_{0}, t_{0}\right)=1$. Then, by Lemma 3.9(i), we have

$$
\Delta^{\alpha} y(t)=\Delta^{\alpha} \widetilde{e}_{p}\left(t, t_{0}\right)=p(t) \widetilde{e}_{p}\left(t, t_{0}\right)=p(t) y(t) .
$$

It follows that

Theorem 5.1 Let $p \in \mathcal{R}_{\alpha}$ and $t_{0} \in \mathbb{T}$. Then $\widetilde{e}_{p}\left(\cdot, t_{0}\right)$ is a solution of the initial value problem

$$
\Delta^{\alpha} y(t)=p(t) y(t), \quad y\left(t_{0}\right)=1
$$

on $\mathbb{T}$.

Theorem 5.2 (Variation of constants) Let $p \in \mathcal{R}_{\alpha}, t_{0} \in \mathbb{T}$, and $y_{0} \in \mathbb{R}$. The unique solution of the initial value problem

$$
\Delta^{\alpha} y=p(t) y+f(t), \quad y\left(t_{0}\right)=y_{0}
$$

is given by

$$
y(t)=y_{0} \widetilde{e}_{p}\left(t, t_{0}\right)+\int_{t_{0}}^{t} \widetilde{e}_{p}(t, \sigma(\tau)) f(\tau) \Delta_{\alpha} \tau, \quad t \in \mathbb{T} .
$$


Proof Let $y$ be given in (21). Using Lemma 3.9(i) and Lemma 4.5,

$$
\begin{aligned}
\Delta^{\alpha} y(t) & =y_{0} \Delta^{\alpha}\left[\widetilde{e}_{p}\left(t, t_{0}\right)\right]+\Delta^{\alpha} \int_{t_{0}}^{t} \widetilde{e}_{p}(t, \sigma(\tau)) f(\tau) \Delta_{\alpha} \tau \\
& =y_{0} p(t) \widetilde{e}_{p}\left(t, t_{0}\right)+\int_{t_{0}}^{t} p(t) \widetilde{e}_{p}(t, \sigma(\tau)) f(\tau) \Delta_{\alpha} \tau+\widetilde{e}_{p}(\sigma(t), \sigma(t)) f(t) \\
& =p(t)\left[y_{0} \widetilde{e}_{p}\left(t, t_{0}\right)+\int_{t_{0}}^{t} \widetilde{e}_{p}(t, \sigma(\tau)) f(\tau) \Delta_{\alpha} \tau\right]+f(t) \\
& =p(t) y(t)+f(t),
\end{aligned}
$$

as desired.

Theorem 5.3 (Variation of constants) Let $p \in \mathcal{R}_{\alpha}, t_{0} \in \mathbb{T}$, and $y_{0} \in \mathbb{R}$. The unique solution of the initial value problem

$$
\Delta^{\alpha} y=\left[\kappa_{1}(\alpha, t)-p(t)\right] y^{\sigma}+f(t), \quad y\left(t_{0}\right)=y_{0}
$$

is given by

$$
y(t)=y_{0} \widetilde{e}_{\kappa_{1} \ominus \alpha p}\left(t, t_{0}\right)+\int_{t_{0}}^{t} \widetilde{e}_{\kappa_{1} \ominus \alpha p}(t, \tau) f(\tau) \Delta_{\alpha} \tau, \quad t \in \mathbb{T} .
$$

Proof Using the conformable product rule, we notice that

$$
\begin{aligned}
\Delta^{\alpha}\left[\widetilde{e}_{p}\left(\cdot, t_{0}\right)\right](t) & =\left[\Delta^{\alpha} y(t)-\left(\kappa_{1}(\alpha, t)-p(t)\right) y^{\sigma}(t)\right] \widetilde{e}_{p}\left(t, t_{0}\right) \\
& =f(t) \widetilde{e}_{p}\left(t, t_{0}\right)
\end{aligned}
$$

Multiplying both sides by $\widetilde{e}_{0}(t, \sigma(\tau))$ and integrating them yields via Lemma 4.4(i)

$$
\begin{aligned}
& \left.y(\tau) \widetilde{e}_{p}\left(\tau, t_{0}\right) \widetilde{e}_{0}(t, \sigma(\tau))\right|_{\tau=t_{0}} ^{t}=\int_{t_{0}}^{t} f(\tau) \widetilde{e}_{p}\left(\tau, t_{0}\right) \widetilde{e}_{0}(t, \sigma(\tau)) \Delta_{\alpha} \tau, \\
& y(t)=y\left(t_{0}\right) \frac{\widetilde{e}_{0}\left(t, \sigma\left(t_{0}\right)\right)}{\widetilde{e}_{p}\left(t, t_{0}\right) \widetilde{e}_{0}(t, \sigma(t))}+\int_{t_{0}}^{t} f(\tau) \frac{\widetilde{e}_{p}\left(\tau, t_{0}\right) \widetilde{e}_{0}(t, \sigma(\tau))}{\widetilde{e}_{p}\left(t, t_{0}\right) \widetilde{e}_{0}(t, \sigma(t))} \Delta_{\alpha} \tau .
\end{aligned}
$$

Now, by Theorem 3.8 (ii) and (13), we have that

$$
\frac{\widetilde{e}_{0}\left(t, \sigma\left(t_{0}\right)\right)}{\widetilde{e}_{p}\left(t, t_{0}\right) \widetilde{e}_{0}(t, \sigma(t))}=\widetilde{e}_{\kappa_{1} \ominus \alpha p}\left(t, t_{0}\right) \quad \text { and } \quad \frac{\widetilde{e}_{p}\left(\tau, t_{0}\right) \widetilde{e}_{0}(t, \sigma(\tau))}{\widetilde{e}_{p}\left(t, t_{0}\right) \widetilde{e}_{0}(t, \sigma(t))}=\widetilde{e}_{\kappa_{1} \ominus_{\alpha} p}(t, \tau) \text {. }
$$

Hence, the assertion follows.

Now, we consider Gronwall's inequality on time scales. We start with a comparison theorem.

Theorem 5.4 Let $y, f \in C_{r d}(\mathbb{T})$ and $p \in \mathcal{R}_{\alpha}^{+}$. Then

$$
\Delta^{\alpha} y(t) \leq p(t) y(t)+f(t) \quad \text { for all } t \in \mathbb{T}
$$


implies

$$
y(t) \leq y\left(t_{0}\right) \widetilde{e}_{p}\left(t, t_{0}\right)+\int_{t_{0}}^{t} \widetilde{e}_{p}(t, \sigma(\tau)) f(\tau) \Delta_{\alpha} \tau \quad \text { for all } t \in \mathbb{T} .
$$

Proof We use the conformable product rule, Lemma 2.10(ii), and Lemma 3.9(ii) to calculate

$$
\begin{aligned}
& \Delta^{\alpha}\left[\widetilde{y e}_{\kappa_{1} \ominus \alpha p}\left(\cdot, t_{0}\right)\right](t) \\
& =\Delta^{\alpha} y(t) \widetilde{e}_{\kappa_{1} \ominus_{\alpha} p}\left(\sigma(t), t_{0}\right)+y(t)\left[\kappa_{1}(\alpha, t)-p(t)\right] \widetilde{e}_{\kappa_{1} \ominus_{\alpha} p}\left(\sigma(t), t_{0}\right) \\
& -\kappa_{1}(\alpha, t) y(t) \widetilde{e}_{\kappa_{1} \ominus_{\alpha} p}\left(\sigma(t), t_{0}\right) \\
& =\left[\Delta^{\alpha} y(t)-p(t) y(t)\right] \widetilde{e}_{\kappa_{1} \ominus \alpha p}\left(\sigma(t), t_{0}\right) \text {. }
\end{aligned}
$$

Since $\kappa_{1} \ominus_{\alpha} p \in \mathcal{R}_{\alpha}^{+}$, we have $\widetilde{e}_{\kappa_{1} \ominus_{\alpha} p}>0$ by Lemma 3.7. Multiplying both sides by $\widetilde{e}_{0}(t, \sigma(\tau))$ and integrating them yields via Lemma 4.4(i)

$$
\begin{aligned}
& \left.y(\tau){\widetilde{e_{1} \ominus_{\alpha} p}}\left(\tau, t_{0}\right) \widetilde{e}_{0}(t, \sigma(\tau))\right|_{\tau=t_{0}} ^{t} \\
& =\int_{t_{0}}^{t}\left[\Delta^{\alpha} y(\tau)-p(\tau) y(\tau)\right] \widetilde{e}_{\kappa_{1} \ominus_{\alpha} p}\left(\sigma(\tau), t_{0}\right) \widetilde{e}_{0}(t, \sigma(\tau)) \Delta_{\alpha} \tau, \\
& y(t) \widetilde{e}_{\kappa_{1} \ominus \alpha p}\left(t, t_{0}\right) \widetilde{e}_{0}(t, \sigma(t))-y\left(t_{0}\right) \widetilde{e}_{0}\left(t, \sigma\left(t_{0}\right)\right) \leq \int_{t_{0}}^{t} f(\tau) \widetilde{e}_{\kappa_{1} \ominus_{\alpha} p}\left(\sigma(\tau), t_{0}\right) \widetilde{e}_{0}(t, \sigma(\tau)) \Delta_{\alpha} \tau, \\
& y(t) \leq y\left(t_{0}\right) \frac{\widetilde{e}_{0}\left(t, \sigma\left(t_{0}\right)\right)}{\widetilde{e}_{\kappa_{1} \ominus \alpha p}\left(t, t_{0}\right) \widetilde{e}_{0}(t, \sigma(t))}+\int_{t_{0}}^{t} \frac{\widetilde{e}_{\kappa_{1} \ominus \alpha p}\left(\sigma(\tau), t_{0}\right) \widetilde{e}_{0}(t, \sigma(\tau))}{\widetilde{e}_{\kappa_{1} \ominus \alpha p}\left(t, t_{0}\right) \widetilde{e}_{0}(t, \sigma(t))} f(\tau) \Delta_{\alpha} \tau .
\end{aligned}
$$

Now, by Theorem 3.8 (i) and (ii), we have that

$$
\begin{aligned}
& \frac{\widetilde{e}_{0}\left(t, \sigma\left(t_{0}\right)\right)}{{\widetilde{e_{k} \ominus_{\alpha} p}}_{\kappa_{1}}\left(t, t_{0}\right) \widetilde{e}_{0}(t, \sigma(t))}=\frac{\widetilde{e}_{p}\left(t, t_{0}\right) \widetilde{e}_{0}(\sigma(t), t)}{\widetilde{e}_{0}\left(t, t_{0}\right) \widetilde{e}_{0}\left(\sigma\left(t_{0}\right), t\right)}=\widetilde{e}_{p}\left(t, t_{0}\right) \quad \text { and } \\
& \frac{{\widetilde{e_{k} \ominus_{\alpha p}}}_{\left(\sigma(\tau), t_{0}\right) \widetilde{e}_{0}(t, \sigma(\tau))}}{\widetilde{e}_{\kappa_{1} \ominus_{\alpha} p}\left(t, t_{0}\right) \widetilde{e}_{0}(t, \sigma(t))}=\left(1-\frac{\kappa_{1}}{\kappa_{0}} \mu\right) \frac{\widetilde{e}_{\kappa_{1} \ominus_{\alpha} p}(\sigma(\tau), t)}{\widetilde{e}_{0}(\sigma(\tau), t)} \\
& =\left(1-\frac{\kappa_{1}}{\kappa_{0}} \mu\right) \widetilde{e}_{p}(t, \sigma(\tau)) \leq \widetilde{e}_{p}(t, \sigma(\tau)) .
\end{aligned}
$$

As a result, the assertion follows.

Theorem 5.5 (Gronwall's inequality) Let $y, f \in C_{r d}, p \in \mathcal{R}_{\alpha}^{+}, p \geq 0$, and $t_{0} \in \mathbb{T}$. Then

$$
y(t) \leq f(t)+\int_{t_{0}}^{t} p(\tau) y(\tau) \widetilde{e}_{0}(t, \sigma(\tau)) \Delta_{\alpha} \tau \quad \text { for all } t \in \mathbb{T}
$$

implies

$$
y(t) \leq f(t)+\int_{t_{0}}^{t} p(\tau) f(\tau) \widetilde{e}_{p}(t, \sigma(\tau)) \Delta_{\alpha} \tau \quad \text { for all } t \in \mathbb{T} .
$$


Proof Define

$$
z(t)=\int_{t_{0}}^{t} p(\tau) y(\tau) \widetilde{e}_{0}(t, \sigma(\tau)) \Delta_{\alpha} \tau,
$$

then $z\left(t_{0}\right)=0$, and by Lemma 4.3 we have

$$
\Delta^{\alpha} z(t)=p(t) y(t) \leq[f(t)+z(t)] p(t)=p(t) z(t)+p(t) f(t) .
$$

By Theorem 5.4, we have

$$
z(t) \leq \int_{t_{0}}^{t} \widetilde{e}_{p}(t, \sigma(\tau)) p(\tau) f(\tau) \Delta_{\alpha} \tau .
$$

Hence, the claim follows because $y(t) \leq f(t)+z(t)$.

Corollary 5.6 Let $y \in \mathbb{C}_{r d}, p \in \mathcal{R}_{\alpha}^{+}$with $p \geq 0$, and $\delta \in \mathbb{R}$. Then

$$
y(t) \leq \delta+\int_{t_{0}}^{t} p(\tau) y(\tau) \widetilde{e}_{0}(t, \sigma(\tau)) \Delta_{\alpha} \tau \quad \text { for all } t \in \mathbb{T}
$$

implies

$$
y(t) \leq \delta \Omega_{p}\left(t, t_{0}\right)+\delta \int_{t_{0}}^{t} \kappa_{1}(\alpha, t) \widetilde{e}_{p}(t, \sigma(\tau)) \Delta_{\alpha} \tau \quad \text { for all } t \in \mathbb{T}
$$

where

$$
\Omega_{p}\left(t, t_{0}\right)=1+\widetilde{e}_{p}\left(t, t_{0}\right) \widetilde{e}_{0}\left(t_{0}, \sigma\left(t_{0}\right)\right)-\widetilde{e}_{0}(t, \sigma(t)) .
$$

Proof Note that

$$
\widetilde{e}_{p}(t, \sigma(\tau))=\widetilde{e}_{\kappa_{1} \ominus_{\alpha} p}(\sigma(\tau), t) \widetilde{e}_{0}(t, \sigma(\tau) .
$$

Hence,

$$
\begin{aligned}
p(t) \widetilde{e}_{p}(t, \sigma(\tau)) & =\kappa_{1}(\alpha, t) \widetilde{e}_{p}(t, \sigma(\tau))-\left[\kappa_{1}(\alpha, t)-p(t)\right] \widetilde{e}_{\kappa_{1} \ominus_{\alpha} p}(\sigma(\tau), t) \widetilde{e}_{0}(t, \sigma(\tau) \\
& =\kappa_{1}(\alpha, t) \widetilde{e}_{p}(t, \sigma(\tau))-\Delta^{\alpha}\left[\widetilde{e}_{\kappa_{1} \ominus_{\alpha} p}(\tau, t)\right] \widetilde{e}_{0}(t, \sigma(\tau) .
\end{aligned}
$$

If we let $f(t)=\delta$ in Theorem 5.5 , then by (23) we have

$$
\begin{aligned}
y(t) & \leq \delta\left[1+\int_{t_{0}}^{t} p(\tau) \widetilde{e}_{p}(t, \sigma(\tau)) \Delta_{\alpha} \tau\right] \\
& =\delta\left[1-\int_{t_{0}}^{t} \Delta^{\alpha}\left[\widetilde{e}_{\kappa_{1} \ominus_{\alpha} p}(\tau, t)\right] \widetilde{e}_{0}\left(t, \sigma(\tau) \Delta_{\alpha} \tau+\int_{t_{0}}^{t} \kappa_{1}(\alpha, t) \widetilde{e}_{p}(t, \sigma(\tau)) \Delta_{\alpha} \tau\right]\right. \\
& =\delta\left[1-\widetilde{e}_{\kappa_{1} \ominus \alpha p}(\tau, t) \widetilde{e}_{0}\left(t,\left.\sigma(\tau)\right|_{\tau=t_{0}} ^{t}+\int_{t_{0}}^{t} \kappa_{1}(\alpha, t) \widetilde{e}_{p}(t, \sigma(\tau)) \Delta_{\alpha} \tau\right]\right.
\end{aligned}
$$




$$
\begin{aligned}
& =\delta\left[1-\left[\widetilde{e}_{0}\left(t, \sigma(t)-\widetilde{e}_{\kappa_{1} \ominus \alpha p}\left(t_{0}, t\right) \widetilde{e}_{0}\left(t, \sigma\left(t_{0}\right)\right)+\int_{t_{0}}^{t} \kappa_{1}(\alpha, t) \widetilde{e}_{p}(t, \sigma(\tau)) \Delta_{\alpha} \tau\right]\right.\right. \\
& =\delta\left[1+\widetilde{e}_{p}\left(t, t_{0}\right) \widetilde{e}_{0}\left(t_{0}, \sigma\left(t_{0}\right)\right)-\widetilde{e}_{0}(t, \sigma(t))\right]+\delta \int_{t_{0}}^{t} \kappa_{1}(\alpha, t) \widetilde{e}_{p}(t, \sigma(\tau)) \Delta_{\alpha} \tau .
\end{aligned}
$$

We note that, for all right-dense points $t \in \mathbb{T}$, we have

$$
\Omega_{p}\left(t, t_{0}\right)=1+\widetilde{e}_{p}\left(t, t_{0}\right) \widetilde{e}_{0}\left(t_{0}, t_{0}\right)-\widetilde{e}_{0}(t, t)=e_{p}\left(t, t_{0}\right)
$$

hence it gives the result obtained in [1] (Corollary 6.4).

\section{Acknowledgements}

The author would like to thank the reviewers and the editors for their valuable suggestions and comments.

\section{Funding}

None.

Availability of data and materials

Not applicable.

\section{Competing interests}

The author declares that there is no conflict of interests regarding the publication of this paper.

\section{Authors' contributions}

The author read and approved the final manuscript.

\section{Publisher's Note}

Springer Nature remains neutral with regard to jurisdictional claims in published maps and institutional affiliations.

Received: 9 May 2019 Accepted: 12 August 2019 Published online: 23 August 2019

\section{References}

1. Anderson, D.R., Ulness, D.J.: Newly defined conformable derivatives. Adv. Dyn. Syst. Appl. 10(2), 109-137 (2015)

2. Benkhettou, N., Hassani, S.: Torres, D.F.M., A conformable fractional calculus on arbitrary time scales. J. King Saud Univ., Sci. 28(1), 93-98 (2016)

3. Zhao, D.-F., You, X.-X.: A new fractional derivative on time scales. Adv. Appl. Math. Anal. 11(1), 1-9 (2016)

4. Hammad, M.A., Khalil, R.: Conformable fractional heat differential equations. Int. J. Pure Appl. Math. 94(2), 215-221 (2014)

5. Chung, W.S.: Fractional Newton mechanics with conformable fractional derivative. J. Comput. Appl. Math. 290 150-158 (2015)

6. Zhao, D.-F., You, X.-X., Cheng, J.: Remarks on conformable fractional derivative on time scales. Adv. Theor. Appl. Math. $11(1), 61-68(2016)$

7. Gulsen, T., Yilmaz, E., Goktas, S.: Conformable fractional Dirac system on time scales. J. Inequal. Appl. 2017, 161 (2017)

8. Gulsen, T., Yilmaz, E., Kemaloglu, H.: Conformable fractional Sturm-Liouville equation and some existence results on time scales. Turk. J. Math. 42, 1348-1360 (2018)

9. Zhao, D.-F., Li, T.: On conformable delta fractional calculus on time scales. J. Math. Comput. Sci. 16, 324-335 (2016)

10. Bohner, M., Peterson, A.: Dynamic Equations on Time Scales: An Introduction with Applications. Birkhäuser, Boston (2001)

11. Bohner, M., Peterson, A.: Advances in Dynamic Equations on Time Scales. Birkhäuser, Boston (2003)

12. Agarwal, R.P., Bohner, M., O’Regan, D., Peterson, A.: Dynamic equations on time scales. J. Comput. Appl. Math. $141(1-2), 1-26(2002)$

13. Aulbach, B., Hilger, S.: A Unified Approach to Continuous and Discrete Dynamics, vol. 184. Inst. für Mathematik (1988)

14. Hilger, S.: Analysis on measure chain-a unified approach to continuous and discrete calculus. Results Math. 18, 18-56 (1990) 